\title{
NORMAL SUBGROUPS CONTAINED IN THE FRATTINI SUBGROUP. II
}

\author{
W. MACK HILI.
}

ABSTRACT. If $p$ is an odd prime and $H$ is a $p$-group with a characteristic subgroup $K$ such that $|K|>|K \cap Z(H)|=p$, then $H$ cannot be a normal subgroup contained in the Frattini subgroup of any finite group $G$.

We consider only finite groups. The order of the group $G$ is $|G|, Z(G)$ is the center of $G, A(G)$ is the automorphism group of $G$ and $I(G)$ is the group of inner automorphisms. If $G$ is nilpotent, $\operatorname{cl}(G)$ denotes its nilpotence class. Other notation is also standard.

Our aim is to prove the following

Theorem. Let $H$ be a p-group, $p$ an odd prime, with a characteristic subgroup $K$ such that $|K|>|K \cap Z(H)|=p$. Then $H$ cannot be a normal subgroup contained in the Frattini subgroup of any finite group G.

This result appears in [6] for arbitrary prime $p$, but under the additional hypothesis that $\operatorname{cl}(K) \neq 2$. It appears in [3] for the case that $p$ is any prime and $G$ is $p$-supersolvable. The case that $|H|=|K|=p^{3}$ is covered in $[5]$.

With no loss of generality (see [6]), we take $K=H$ and $\operatorname{cl}(H)=2$. Then $H$ is extra-special. For a discussion of extra-special $p$-groups and their automorphisms the reader is referred to [1], [7] and [8].

Our argument is based on two lemmas, the first of which is mentioned in [2]. (The author is grateful to Professor David Goldschmidt for a very helpful conversation concerning this result.)

Lemma 1. If $H$ is an extra-special p-group of exponent $p$, $p$ odd, then $A(H)$ splits over $I(H)$.

Proof. $H=\left\langle x_{1}, x_{2}, \ldots, x_{n}, z\right\rangle$ with $x_{i}^{p}=z^{p}=1$ for each $i$ and $\left[x_{1}, x_{2}\right]=\left[x_{3}, x_{4}\right]=\cdots=\left[x_{n-1}, x_{n}\right]=z$. Further, $\left[x_{i}, x_{j}\right]=1$ unless $\{i, j\}$ is one of $\{1,2\},\{3,4\}, \cdots,\{n-1, n\}$. Each element of $H$ has unique representation as $\left(\Pi_{i=1}^{n} x_{i}^{a}\right) z^{b}$ with $0 \leq a_{i}, b<p$.

If $\sigma \in A(H)$, then for each $i, \sigma\left(x_{i}\right)=\left(\operatorname{II}_{j=1}^{n} x_{j}^{a_{i j}}\right)_{z} b_{i}$ with $\left(a_{i j}\right) \in \operatorname{GL}(n, p)$ and $0 \leq b_{i}<p$. Further, $\sigma \in I(H)$ if and only if $\left(a_{i j}\right)$ is the identity matrix.

Received by the editors December 16, 1974.

AMS (MOS) subject classifications (1970). Primary 20D25; Secondary 20D15, $20 \mathrm{D} 45$.

Key words and phrases. Extra-special p-group, Frattini subgroup. 
Now the mapping $\tau$ of $\left\{x_{1}, x_{2}, \cdots, x_{n}, z\right\}$ into $H$, defined by $\tau\left(x_{i}\right)=x_{i}^{-1}$ $(i=1,2, \ldots, n)$ and $r(z)=z$, determines an automorphism $\tau \in A(H)$, and $C_{A(H)}(\tau)$ has trivial intersection with $I(H)$. Let $\sigma$ map $H$ into $H$ and $y$ map $\left\{x_{1}, x_{2}, \ldots, x_{n}\right\}$ into $H$ and suppose that for $i=1,2, \ldots, n$,

$$
\sigma\left(x_{i}\right)=\left(\prod_{j=1}^{n} x_{j}^{a_{i j}}\right) z^{b_{i}} \text { and } \gamma\left(x_{i}\right)=\left(\prod_{j=1}^{n} x_{j}^{a_{i j}}\right) z^{c_{i}} \text {. }
$$

Consider the system of linear congruences

$$
\sum_{j=1}^{n} a_{i j} t_{j} \equiv c_{i}-b_{i} \quad(\bmod p), \quad i=1,2, \ldots, n .
$$

If $\left(a_{i j}\right)$ is nonsingular, there exists a unique solution $\left(d_{1}, d_{2}, \ldots, d_{n}\right)$ with $0 \leq d_{i}<p$. The mapping $\rho$ of $\left\{x_{1}, x_{2}, \ldots, x_{n}, z\right\}$ into $H$ defined by $\rho\left(x_{i}\right)=$ $x_{i} z^{i}(i=1,2, \ldots, n)$ and $\rho(z)=z$ determines an inner automorphism $\rho \epsilon$ $I(H)$ and

$$
\rho \sigma\left(x_{i}\right)=\rho\left[\left(\prod_{j=1}^{n} x_{j}^{a_{i j}}\right) z^{b_{i}}\right]=\left(\prod_{j=1}^{n} x_{j}^{a_{i j}}\right) z^{e^{i}}=\left(\prod_{j=1}^{n} x_{j}^{a_{i j}}\right) z^{c_{i}}=\gamma\left(x_{i}\right)
$$

where $e_{i}=\sum_{j=1}^{n} a_{i j} d_{j}+b_{i}$. In particular, if $\sigma \in A(H)$, then $\gamma$ agrees with $\rho \sigma$ on the generating set $\left\{x_{1}, x_{2}, \ldots, x_{n}\right\}$ and, hence, determines an automorphism $y \in A(H)$ with $\rho \sigma=\gamma$.

We now show that for arbitrary $\tau \in A(H)$, the exponents $c_{i}(i=1,2$, $\cdots, n)$ above can be selected so that $\gamma \in C_{A(H)}(\tau)$. For $i=1,2, \ldots, n$ let $c_{i}$ be the unique solution of the linear congruence

$$
2 t+f_{i}=2 t+\sum_{k=1}^{n-1} a_{i k} a_{i(k+1)} \equiv 0 \quad(\bmod p) .
$$

Then

$$
\begin{aligned}
\gamma \tau\left(x_{i}\right) & =\gamma\left(x_{i}^{-1}\right)=\left(\prod_{j=1}^{n} x_{j}^{a_{i j}}\right)^{-1} z^{-c_{i}}=\left(\prod_{j=1}^{n} x_{j}^{-a_{i j}}\right) z^{-c_{i}-f_{i}} \\
& =\left(\prod_{j=1}^{n} x_{j}^{-a_{i j}}\right) z^{c_{i}}=\tau\left[\left(\prod_{j=1}^{n} x_{j}^{a_{i j}}\right) z^{c_{i}}\right]=\tau \gamma\left(x_{i}\right) .
\end{aligned}
$$

Thus, for each $\sigma \in A(H)$, there exists $\rho \in I(H)$ and $\gamma \in C_{A(H)}(\tau)$ such that $\sigma=\rho^{-1} \gamma$, i.e. $A(H)=I(H) C_{A(H)}(\tau)$. Hence, $C_{A(H)}(\tau)$ complements $I(H)$ in $A(H)$, completing the proof of Lemma 1.

Lemma 2. If $H$ is an extra-special p-group of exponent $p^{2}, p$ odd, then $H$ has a characteristic subgroup $K$ of order $p^{2}$.

Proof. $H=\left\langle x_{1}, x_{2}, \cdots, x_{n}, z\right\rangle$ with $x_{1}^{p^{2}}=x_{i}^{p}=z^{p}=1 \quad(i=2,3$, $\cdots, n), x_{1}^{p}=z$ and $\left[x_{1}, x_{2}\right]=\left[x_{3}, x_{4}\right]=\cdots=\left[x_{n-1}, x_{n}\right]=z$. Further, $\left[x_{i}, x_{j}\right]=1$ unless $\{i, j\}$ is one of $\{1,2\},\{3,4\}, \ldots,\{n-1, n\}$. The subgroup $\left\langle z, x_{2}, \cdots, x_{n}\right\rangle$, consisting precisely of those elements in $H$ satisfying $b^{p}=1$, is characteristic in $H$, and its center, also characteristic in $H$, is 
$\left\langle z, x_{2}\right\rangle$, of order $p^{2}$. Take $K=\left\langle z, x_{2}\right\rangle$. This proves Lemma 2 .

Now, let $H$ be an extra-special $p$-group, $p$ odd. If the exponent of $H$ is $p$, then Lemma 1 together with III. 3.2 and III. 3.13 of [7] implies that $H$ cannot be a normal subgroup contained in the Frattini subgroup of any finite group $G$. If on the other hand the exponent of $H$ is $p^{2}$, then $H$ has a characteristic subgroup $K$ of order $p^{2}$ (Lemma 2), which of necessity intersects $Z(H)$ in a subgroup of order $p$. By [6], the desired conclusion follows, and the proof of the theorem is complete.

For the case $p=2$, we have very little information. Again we lose no generality by taking $H=K$ and $\operatorname{cl}(H)=2$. Thus, as before, $H$ is extraspecial. From (2), the splitting of $A(H)$ over $I(H)$ occurs for extra-special 2 -groups of orders $2^{3}$ and $2^{5}$ and does not occur for those of order $2^{7}$ and larger. Hence, a 2-group $H$ with characteristic subgroup $K$ of order $2^{3}$ or $2^{5}$ and intersecting $Z(H)$ in a subgroup of order 2 cannot be a normal subgroup contained in the Frattini subgroup of any finite group $G$. Since the splitting of $A(H)$ over $I(H)$ is only a sufficient condition for the above nonembeddability conclusion, the question remains open for extra-special 2-groups of larger orders.

Added in proof. Professor Homer Bechtell has observed that Griess' work (2) can be used to show that if $H$ is an extra-special 2-group of order larger than 32, there exists a (nonsolvable) group $G$ having Frattini subgroup $H$.

\section{REFERENCES}

1. D. Gorenstein, Finite groups, Harper \& Row, New York, 1968. MR 38 \#229.

2. R. L. Griess, Jr., Automorphisms of extra special groups and nonvanishing degree 2 cohomology, Pacific J. Math. 48 (1973), 403-422.

3. W. M. Hill, Frattini subgroups of p-closed and p-supersolvable groups, Israel J. Math. 19 (1974), 208-211.

4. - - Extra-special normal p-subgroups, Notices Amer. Math. Soc. 21 (1974), A-526. Abstract \#74T-A214.

5. W. M. Hill and D. B. Parker, The nilpotence class of the Frattini subgroup, Israel J. Math. 15 (1973), 211-215. MR $48 \# 422$.

6. W. M. Hill and C. R. B. Wright, Normal subgroups contained in the Frattini subgroup, Proc. Amer. Math. Soc. 35 (1972), 413-415. MR $46 \# 252$.

7. B. Huppert, Endliche Gruppen. I, Die Grundlehren der math. Wissenschaften, Band 134, Springer-Verlag, Berlin and New York, 1967. MR 47 \#302.

8. D. L. Winter, The automorphism group of an extraspecial p-group, Rocky Monta in J. Math. 2 (1972), 159-168. MR $45 \# 6911$.

DEPARTMENT OF MATHEMATICS, WORCESTER STATE COLLEGE, WORCESTER, MASSACHUSET TS 01602 